Letters to the Editor

\title{
Amperometric Determination of Surfactants Using a Gold Electrode Modified with a Self-Assembled Monolayer of Stearylthiol
}

\author{
Tetsuo Kawaguchi, Yuji Yamauchi, Hatsuo Maeda and Hidenobu Ohmori \\ Faculty of Pharmaceutical Sciences, Osaka University, Yamadaoka, Suita, Osaka 565, Japan
}

Keywords Surfactant, amperometric sensor, strearylthiol, monolayer, electrode modification

Surfactants, which find many applications as emulsifiers and solubilizers, are also environmental pollutants. Considerable attention has thus been focused on their detection and/or determination. ${ }^{1}$ Highperformance liquid chromatography (HPLC) has been recommended as an effective technique for this purpose. However, except for certain surfactants with characteristic UV absorption ${ }^{2}$ or fluorescence ${ }^{3}$, the detection of most surfactants by HPLC has been performed with refractive-index detectors ${ }^{4}$ whose sensitivity and selectivity are not always satisfactory. Although a postcolumn detector based on ion-pair extraction was shown to give reasonable results ${ }^{5}$, a simpler and more facile method seems to be worthy of investigation.

We recently reported that the structure of a LangmuirBlodgett (LB) membrane cast on a glassy carbon plate is distorted by surfactants to change its permeability to $\mathrm{Fe}(\mathrm{CN})_{6}{ }^{3-}$ used as a marker ion. ${ }^{6}$ This finding suggested the possibility of electrochemical detection of surfactants by a solid electrode modified with a lipid membrane. LB membranes, however, are too fragile to be utilized in the flow system of an HPLC detector. On the other hand, alkanethiols form stable self-assembled monolayers (SAMs) on a gold surface. 7,8 A novel electrochemical detection of surfactants could thus be attained if the permeability of an SAM to marker ions is affected by surfactants in a manner similar to that of an LB membrane. We discuss in this communication how the electrochemical response of an SAM-coated gold electrode towards marker ions, $\mathrm{Fe}(\mathrm{CN})_{6}{ }^{3-}$ and $\mathrm{Fe}^{3+}$, depends on the structure of the surfactants as well as their concentrations.

The SAM-coated electrode was prepared as follows. A gold disk electrode $(1.6 \mathrm{~mm} \phi)$ polished with $0.05 \mu \mathrm{m}$ alumina was treated by multiple triangular potential sweeps $\left(-0.2-+1.8 \mathrm{~V}\right.$ vs. $\left.\mathrm{Ag} / \mathrm{AgCl}, 10 \mathrm{~V} \mathrm{~s}^{-1}\right)$ in $1 \mathrm{M}$ $\mathrm{H}_{2} \mathrm{SO}_{4}$ for $10 \mathrm{~min}$. After being rinsed with distilled water, the electrode was immersed in an ethanolic solution of $10 \mathrm{mM}$ stearylthiol $\left(\mathrm{CH}_{3}\left(\mathrm{CH}_{2}\right)_{17} \mathrm{SH}\right)$ for $10 \mathrm{~h}$. The thiol-coated electrode was then washed by keeping it in ethanol for $\mathbf{3 0} \mathrm{min}$ followed by air-drying.

The amperometric response of the SAM-coated electrode to the marker ions $(5 \mathrm{mM})$ in aqueous $\mathrm{KCl}$ $(0.1 \mathrm{M})$ was examined by measuring the steady-state reduction currents $\left(I\right.$ and $\left.I^{0}\right)$ due to the marker ions at $0.0 \mathrm{~V} v$ s. $\mathrm{Ag} / \mathrm{AgCl}$, where $I$ refers to the current in the presence and $I^{0}$ to that in the absence of the surfactants. The $I^{0}$ values were 10 and $50 \mathrm{nA}$ for $\mathrm{Fe}(\mathrm{CN})_{6}{ }^{3-}$ and $\mathrm{Fe}^{3+}$, respectively. Figures 1 and 2 show the dependence of the current ratio $\left(I / I^{0}\right)$ on the concentration of surfactants with hydrophobic $\mathrm{C}-12$ alkyl chain, $\mathrm{CH}_{3}-$ $\left(\mathrm{CH}_{2}\right)_{11} \mathrm{~N}\left(\mathrm{CH}_{3}\right)_{3} \mathrm{Br}$ (1), $\mathrm{CH}_{3}\left(\mathrm{CH}_{2}\right)_{11} \mathrm{SO}_{4} \mathrm{Na}$ (2), $\mathrm{CH}_{3-}$ $\left(\mathrm{CH}_{2}\right)_{11}\left(\mathrm{OCH}_{2} \mathrm{CH}_{2}\right)_{23} \mathrm{OH}$ (3), and $\mathrm{CH}_{3}\left(\mathrm{CH}_{2}\right)_{11} \mathrm{OH}$ (4). When $\mathrm{Fe}(\mathrm{CN})_{6}{ }^{3-}$ was employed as the marker, an increase in the $I / I^{0}$ value with the concentration was observed for only the cationic surfactant 1 (Fig. 1). A reverse behavior was noted with the cationic marker $\left(\mathrm{Fe}^{3+}\right)$ : the current ratio increased in the presence of the anionic surfactant 2 (Fig. 2). In the case of an unmodified gold electrode, the effect of any of surfactants 1-4 on the current ratio was essentially negligible. Since the coexistence of a surfactant with a charge opposite to that of the marker ion causes an increase in the current ratio, it is suggested that an ion-

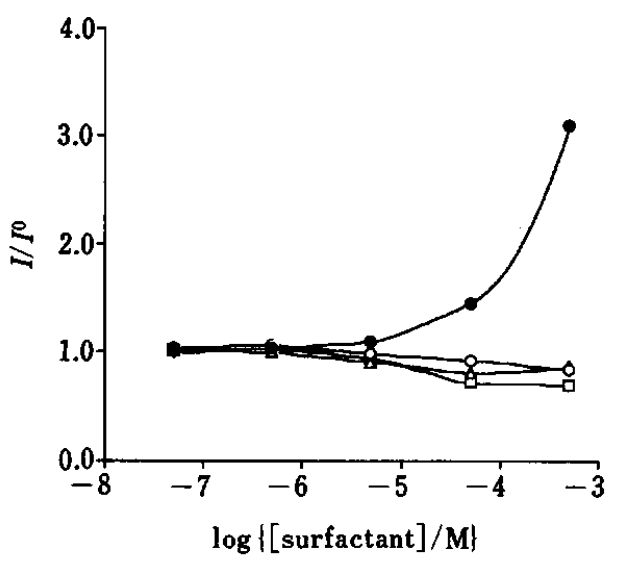

Fig. 1 Effects of surfactants with a hydrophobic C-12 alkyl chain on the amperometric response of an SAM-coated gold electrode to $\mathrm{Fe}(\mathrm{CN})_{6}{ }^{3-}$ in aqueous $\mathrm{KCl}(0.1 \mathrm{M})$ containing $\mathrm{K}_{3} \mathrm{Fe}(\mathrm{CN})_{6}(5 \mathrm{mM})$. O, surfactant $1 ; 0,2 ; \Delta, 3 ; \square, 4$. 


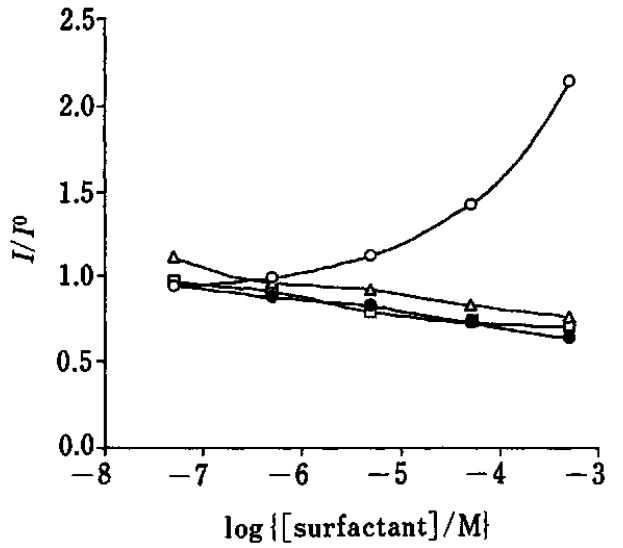

Fig. 2 Effects of surfactants with a hydrophobic C-12 alkyl chain on the amperometric response of an SAM-coated gold electrode to $\mathrm{Fe}^{3+}$ in aqueous $\mathrm{KCl}(0.1 \mathrm{M})$ containing $\mathrm{FeCl}_{3}$ (5 mM). $\quad$, surfactant $1 ; O, 2 ; \Delta, 3, \square, 4$.

pair is formed between the two species to facilitate access of the latter ion to the electrode surface from the aqueous phase through the SAM. The origin of the observed phenomenon can thus be considered to be similar to that of ion-pair extraction. When the charge of the surfactant was zero, or identical to that of the marker ion, the current ratio gradually decreased with the surfactant concentration. These results indicate that all of the surfactants will penetrate into the SAM and, except for those charged oppositely to the marker ion, will hamper access of the marker ion to the electrode surface.

In order to determine the possibility of applying the present results to the determination of surfactants, the dependence of the current ratio on the surfactant concentration was investigated. As can be seen in Fig. 3, linear relationship were obtained between the logarithms of the two variables for the reduction of $\mathrm{Fe}(\mathrm{CN})_{6}{ }^{3-}$ in the presence of one of the three cationic surfactants ${ }^{9}, 1, \mathrm{CH}_{3}\left(\mathrm{CH}_{2}\right)_{9} \mathrm{~N}\left(\mathrm{CH}_{3}\right)_{3} \mathrm{Br}$ (5), and $\mathrm{CH}_{3}-$ $\left(\mathrm{CH}_{2}\right)_{13} \mathrm{~N}\left(\mathrm{CH}_{3}\right)_{3} \mathrm{Br}(6)$. The concentration at which the straight line intersects the abscissa decreases with an increase in the alkyl chain length of the surfactants, indicating that an increase in the hydrophobicity of the surfactant results in an increase in the number of ionpairs transferring into the SAM.

The SAM-coated electrode employed can be readily prepared with reasonable stability and reproducibility to repeated measurements, and will be a convenient tool for the determination of surfactants. A restriction of the

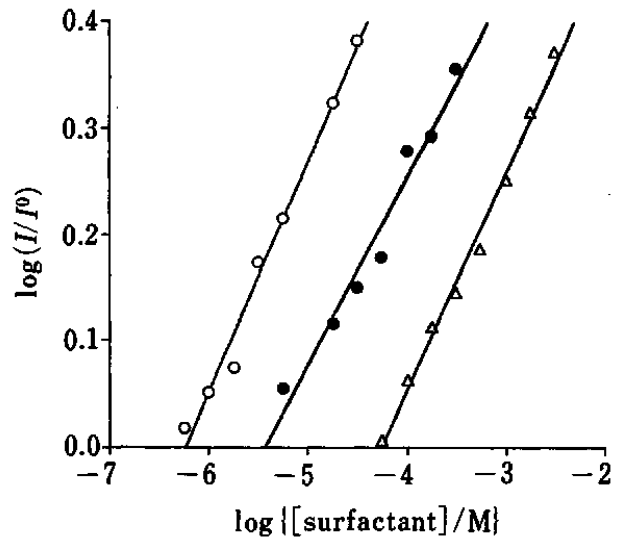

Fig. 3 Logarithmic plots of the current ratio as a function of the surfactant concentration in aqueous $\mathrm{KCl}(0.1 \mathrm{M})$ containing $\mathrm{K}_{3} \mathrm{Fe}(\mathrm{CN})_{6}(5 \mathrm{mM})$. $\quad$, surfactant $1 ; \Delta, 5 ; 0,6$.

present method can be encountered with samples contaminated by thiols and/or disulfides, which would strongly interact with the gold electrode to give erroneous results. The application of the SAM-coated electrode as a detector of surfactants in an HPLC system is in progress.

\section{References and Notes}

1. Y. Suzuki and K. Tsuji, Bunseki, 1989, 121.

2. A. Marcomini and W. Giger, Anal. Chem., 59, 1709 (1987).

3. A. Nakae, K. Tsuji and M. Yamanaka, Anal. Chem., 52, 2275 (1980).

4. K. Nakamura and Y. Morikawa, J. Am. Oil Chem. Soc., 61, 1130 (1984).

5. M. Kanesato, K. Nakamura, O. Nakata and Y. Morikawa, J. Am. Oil Chem. Soc., 64, 434 (1987).

6. T. Kawaguchi, Y. Yamauchi, H. Maeda and H. Ohmori, Chem. Pharm. Bull., 40, 1080 (1992).

7. M. D. Porter, T. B. Bright, D. L. Allara and C. E. D. Chidsey, J. Am. Chem. Soc., 109, 3559 (1987).

8. C. D. Bain, E. B. Troughton, Yu-T. Tao, J. Evall, G. M. Whitesides and R. G. Nuzzo, J. Am. Chem. Soc., 111, 321 (1989).

9. At present, the reason for the logarithmic correlation of the two variables is not known, but should be explored further.

(Received May 21, 1992)

(Accepted July 14, 1992) 